\title{
Assaying macrophage activity in a murine model of inflammatory bowel disease using fluorine-19 MRI
}

\author{
Deepak K Kadayakkara', Sarangarajan Ranganathan ${ }^{2}$, Won-Bin Young ${ }^{3}$ and Eric T Ahrens ${ }^{1}$
}

\begin{abstract}
Macrophages have an important role in the pathogenesis of most chronic inflammatory diseases. A means of noninvasively quantifying macrophage migration would contribute significantly towards our understanding of chronic inflammatory processes and aid the evaluation of novel therapeutic strategies. We describe the use of a perfluorocarbon tracer reagent and in vivo ${ }^{19} \mathrm{~F}$ magnetic resonance imaging (MRI) to quantify macrophage burden longitudinally. We apply these methods to evaluate the severity and three-dimensional distribution of macrophages in a murine model of inflammatory bowel disease (IBD). MRI results were validated by histological analysis, immunofluorescence and quantitative real-time polymerase chain reaction. Selective depletion of macrophages in vivo was also performed, further validating that macrophage accumulation of perfluorocarbon tracers was the basis of ${ }^{19} \mathrm{~F} M R I$ signals observed in the bowel. We tested the effects of two common clinical drugs, dexamethasone and cyclosporine A, on IBD progression. Whereas cyclosporine A provided mild therapeutic effect, unexpectedly dexamethasone enhanced colon inflammation, especially in the descending colon. Overall, ${ }^{19} \mathrm{~F}$ MRI can be used to evaluate early-stage inflammation in IBD and is suitable for evaluating putative therapeutics. Due to its high macrophage specificity and quantitative ability, we envisage ${ }^{19} \mathrm{~F}$ MRI having an important role in evaluating a wide range of chronic inflammatory conditions mediated by macrophages.

Laboratory Investigation (2012) 92, 636-645; doi:10.1038/labinvest.2012.7; published online 13 February 2012
\end{abstract}

KEYWORDS: bowel; inflammation; macrophage; MRI; perfluorocarbon

Inflammatory bowel disease (IBD) such as Crohn's disease and ulcerative colitis are premalignant conditions that significantly increase the risk of colorectal cancer. ${ }^{1}$ The degree of risk of colorectal cancer depends on the anatomical extent, duration and age of onset of IBD. Computed assisted tomography and magnetic resonance imaging (MRI) are non-invasive imaging techniques increasingly being used for evaluation of IBD, ${ }^{2}$ and the diagnosis is commonly confirmed by colonoscopic biopsy. ${ }^{3}$ Colonoscopic biopsy is invasive and requires multiple tissue bites for diagnosis and may cause patient discomfort. Longitudinal, non-invasive quantification of bowel inflammation could potentially provide substantial benefits to the diagnosis and therapeutic monitoring of IBD and the development of new therapeutics.

Resident macrophages in the colon have a key role in the homeostasis of the bowel, ${ }^{4,5}$ and macrophages derived from blood monocytes are important mediators of chronic inflammation in IBD along with Th1- and Th2-type T cells. ${ }^{4-12}$
Thus, non-invasive quantification of macrophage activity in vivo could potentially predict the extent, severity and treatment response in IBD.

Among non-invasive imaging techniques, MRI is often preferable because it does not involve ionizing radiation, has reasonably high spatial and temporal resolution and has excellent soft tissue contrast. With MRI, bowel inflammation is commonly assessed by measuring wall thickness in $\mathrm{T}_{2}$-weighted and Gd-enhanced $\mathrm{T}_{1}$-weighted images. ${ }^{13}$ However, bowel wall thickness does not differentiate between active and inactive disease, and Gd-induced contrast is nonspecific due to leaky exudation into the bowel wall. Cellular MRI using superparamagnetic iron-oxide nanoparticles, which are taken up by the reticuloendothelial system, has been used to study macrophage burden at inflammatory sites in animal models. ${ }^{14}$ Cellular MRI using iron-oxides has potentially high sensitivity, but its use for IBD can be challenging due to the negative contrast produced by labeled cells,

\footnotetext{
${ }^{1}$ Department of Biological Sciences, Carnegie Mellon University, Pittsburgh, PA, USA; ${ }^{2}$ Department of Pathology, University of Pittsburgh School of Medicine, Children's Hospital of Pittsburgh, One Children's Hospital Drive, Pittsburgh, PA, USA and ${ }^{3}$ Department of Radiology, University of Pittsburgh School of Medicine, Pittsburgh, PA, USA

Correspondence: Dr ET Ahrens, PhD, Department of Biological Sciences, Carnegie Mellon University, 4400 Fifth Avenue 15213, Pittsburgh, PA 15213, USA.

E-mail: eta@andrew.cmu.edu

Received 9 September 2011; revised 29 November 2011; accepted 20 December 2011
} 
the high background ${ }^{1} \mathrm{H}$ signal and pronounced intrinsic contrast in bowel and abdominal regions. Moreover, the relationship between signal intensity and concentration of contrast agents is non-linear, making quantitative analyses of disease burden from images challenging.

${ }^{19} \mathrm{~F}$ MRI of inflammation using perfluorocarbon (PFC) 'tracer' emulsions is an emerging approach that yields positive contrast images and has been used to detect inflammatory loci in animal models. ${ }^{15-19}$ Due to the absence of detectable endogenous ${ }^{19} \mathrm{~F}$ in biological tissues, there is no background, and inflammatory foci appear hyperintense in spin density-weighted images. In addition, ${ }^{19} \mathrm{~F}$ MRI signal can be readily quantified by integrating the signal in regions of interest (ROIs), which is linearly proportional to the macrophage burden. ${ }^{20}$

Interleukin-10 $0^{-1-}\left(\mathrm{IL}-10^{-1-}\right)$ mice develop colitis with a pathology that closely resembles human IBD in disease manifestation and course. ${ }^{21-23}$ As observed in human IBD, IL-10 $0^{-1-}$ mice require intestinal bacteria to develop IBD. Mice develop a progressive chronic inflammation that ultimately may progress into colitis-associated colon cancer. IL-10 gene polymorphism has been implicated in human IBD in several studies..$^{24,25}$ IBD in IL-10 $0^{-1-}$ mice run a variable course that can be synchronized by administration of piroxicam. ${ }^{26}$ Macrophages are necessary mediators of inflammation in murine IBD. In IL- $10^{-/-}$mice, elimination of intestinal macrophages reduces the development of colitis; ${ }^{27}$ a similar function for macrophages was observed in other mouse models of IBD such as dextran sulphate sodium (DSS)-induced colitis. ${ }^{28}$

In this study, we evaluated the use of ${ }^{19} \mathrm{~F}$ cellular MRI using PFC emulsion to detect and quantify the severity and extent of bowel inflammation in vivo. These studies utilized a piroxicam-induced IBD model in IL- $10^{-/-}$mice. Using confocal immunofluorescence microscopy and quantitative real-time PCR (qRT-PCR) of macrophage RNA, we demonstrate that macrophage activity in the colon forms the basis of ${ }^{19} \mathrm{~F}$ signals detected in the colon. This was further confirmed by selectively ablating macrophages in vivo and examining the consequences via ${ }^{19} \mathrm{~F}$ MRI and immunofluorescence. We then examined the ability of ${ }^{19} \mathrm{~F}$ MRI to detect the efficacy of putative IBD therapeutics, including dexamethasone and cyclosporine A (CsA). Our studies confirm that ${ }^{19} \mathrm{~F}$ MRI is an effective and sensitive non-invasive technique for quantifying early stages of inflammation in IBD and monitoring treatment response. Overall, we believe that these methodologies will be useful for many other inflammatory diseases and malignancies where macrophages have an important role.

\section{MATERIALS AND METHODS Mouse Model of IBD}

Animal protocols were approved by the Carnegie Mellon University IACUC. All mice received humane care in compliance with the Guide for the Care and Use of Laboratory
Animals, published by the NIH. Transgenic IL- $10^{-/-}$mice on C57/Bl6 background were purchased from Jackson laboratories (Bar Harbor, ME, USA). Female 9-12-week-old mice $(n=22)$ were fed with piroxicam-doped chow $(220 \mathrm{ppm})$ for 14 days to synchronize IBD induction. To detect early bowel irritation, mice were tested for the presence of occult blood in stool using a hemoccult test (Hemoccult Sensa, Beckman Coulter, Brea, CA, USA). After 2 weeks, the piroxicam diet was discontinued, and mice were placed on normal chow. Animal weights were monitored daily throughout the entire study period.

\section{IBD Treatment}

The first treatment group $(n=5)$ received clodronate liposomes delivered intraperitoneally $(0.1 \mathrm{ml}$ per dose). Clodronate was a gift from Roche Diagnostics $\mathrm{GmbH}$, Mannheim, Germany, and was formulated into liposomes using methods previously described. ${ }^{29}$ The first dose was administered 2 days prior to piroxicam treatment, followed by two additional doses separated by 5 days after the start of piroxicam exposure. The second treatment group $(n=5)$ received dexamethasone intraperitoneally $(1.2 \mathrm{mg} / \mathrm{kg})$ daily for 2 weeks. A third treatment group $(n=4)$ was administered CsA intraperitoneally $(10 \mathrm{mg} / \mathrm{kg})$ daily for 2 weeks. For both dexamethasone and CsA, the initial doses were administered 2 days prior to piroxicam treatment.

\section{Administration of PFC Emulsion}

On day 15 , mice were injected with $0.2 \mathrm{ml}$ of a commercial PFC emulsion formulation (VS-1000H, Celsense, Pittsburgh, PA, USA) intravenously via the tail vein using a $28 \mathrm{G}$ needle. This emulsion formulation contains $30 \% \mathrm{v} / \mathrm{v}$ perfluoropolyether with a mean emulsion droplet diameter of $145 \mathrm{~nm}$ according to the manufacturer. To aid in post-MRI histological analysis, the PFC emulsion was rendered fluorescent prior to injection via a premix step of the PFC emulsion with a lipophilic dialkylcarbocyanine (DiI, V22885, Molecular Probes, Eugene, OR, USA) fluorophore, as previously described..$^{20,30}$

\section{In Vivo MRI}

Mice were imaged on days 2, 9, 16, 23 and 30 after PFC injection. Imaging was performed using an $11.7 \mathrm{~T}$ vertical bore microimaging system (Bruker Biospin, Billerica, MA, USA). A birdcage volume resonator, tunable to either ${ }^{19} \mathrm{~F}$ or ${ }^{1} \mathrm{H}$ in situ, was utilized. The mice were anesthetized, intubated, placed on a mechanical ventilator, and maintained on $0.75 \%$ isoflurane in $70 \% \mathrm{O}_{2}$ and $30 \% \mathrm{~N}_{2} \mathrm{O}$ inhalation gas during the imaging sessions. Respiratory gating was used to minimize motion artifacts from abdominal motion. The animal's temperature was maintained at $37^{\circ} \mathrm{C}$. A reference capillary tube containing a 1:16 dilution of PFC emulsion was placed along the mouse's left torso. High-resolution ${ }^{1} \mathrm{H}$ images were obtained using a $\mathrm{T}_{2}$-weighted spin echo sequence (parameters: Recycle time (TR)/echo time 
$(\mathrm{TE})=3000 / 40 \mathrm{~ms}$, matrix size $=512 \times 256$, field of view $(\mathrm{FOV})=4.06 \times 2.96 \mathrm{~cm}$, slice thickness $=2 \mathrm{~mm}, \quad \mathrm{NA}=4$, slices $=20$ ). The co-registered ${ }^{19} \mathrm{~F}$ images were obtained using a rapid acquisition with refocused echoes (RARE) sequence (parameters: RARE factor $=8, \mathrm{TR} / \mathrm{TE}=1900 / 10 \mathrm{~ms}$, matrix size $=64 \times 32, \mathrm{FOV}=4.06 \times 2.96 \mathrm{~cm}$, slice thickness $=2 \mathrm{~mm}$, $\mathrm{NA}=64$, slices $=20)$. The ${ }^{19} \mathrm{~F}$ images were rendered in pseudo-color and superimposed onto the ${ }^{1} \mathrm{H}$ images using the Voxel Tracker software program (Celsense). Each image slice was scaled for optimal visualization and presentation.

\section{In Vivo Quantification and Visualization of ${ }^{19} \mathrm{~F}$ Signal in the Bowel}

The in vivo ${ }^{19} \mathrm{~F}$ signal in the bowel was quantified relative to the external PFC reference capillary yielding the apparent number of fluorine atoms along the full length of the bowel wall. The ${ }^{19} \mathrm{~F}$ quantification was performed using built-in features of Voxel Tracker. Using the raw data, ROIs were defined containing ${ }^{19} \mathrm{~F}$ signal in the bowel wall slice-by-slice, and the integrated intensity within the ROI was calculated. Similarly, ROI in the reference capillary and a region containing pure noise (ie, no ${ }^{19} \mathrm{~F}$ signals) were also delineated. Voxel Tracker amalgamates these inputs to calculate the total apparent ${ }^{19} \mathrm{~F}$ for all ROIs in the slice packet of the bowel.

The in vivo ${ }^{19} \mathrm{~F}$ distribution in the bowel was also volumerendered in three-dimensions using Amira software (Visage Imaging, San Diego, CA, USA). Manual thresholding and automatic segmentation was performed on ${ }^{19} \mathrm{~F}$ images using built-in routines. An isosurface-rendering feature was used for building the three-dimensional (3D) visualization. To aid bowel visualization, the ${ }^{19} \mathrm{~F}$ signals from the spleen and liver were digitally removed. The ${ }^{1} \mathrm{H}$ images were rendered in gray scale as orthoslices, and superimposed with the $3 \mathrm{D}{ }^{19} \mathrm{~F}$ isosurface rendering.

\section{Colon Thickness Measurement}

Colon thickness was measured on day 2 post injection using the image display and processing module in Paravision 4.0. software (Bruker Biospin). For each subject, maximum wall thickness of ascending and descending colon was calculated using the interactive distance measurements tool in Paravision. The mean thickness and the s.e.m. were calculated by combining the data obtained from different subjects in each group.

\section{MRI of the Fixed Colon Tissues}

At the completion of the longitudinal study, mice were euthanized, the colon tissue excised, cleaned in phosphatebuffered saline, and then fixed in $4 \%$ paraformaldehyde. The fixed colon tissues were further imaged using extremely highresolution MRI to further elucidate lesion topology along the length of the bowel. A thin glass rod was inserted into the lumen to straighten the fixed colon, and samples were placed into a sealed $15 \mathrm{ml}$ plastic tube containing phosphatebuffered saline doped with $1 \mathrm{mg} / \mathrm{ml}$ of Feridex iron-oxide nanoparticles (AMAG Pharmaceuticals, Lexington, MA, USA) to attenuate the background ${ }^{1} \mathrm{H}$ signal. The sample was placed in a $10-\mathrm{mm}$ diameter birdcage coil and imaged in $3 \mathrm{D}$ at $11.7 \mathrm{~T}$ (Bruker). The $\mathrm{T}_{2}$-weighted ${ }^{1} \mathrm{H}$ images were obtained using a spin echo sequence (parameters: $\mathrm{TR} / \mathrm{TE}=1000$ / $10 \mathrm{~ms}$, matrix size $=512 \times 256, \quad \mathrm{FOV}=3 \times 1.1 \mathrm{~cm}$, slice thickness $=1 \mathrm{~mm}, \quad \mathrm{NA}=4, \quad$ slices $=6) .{ }^{19} \mathrm{~F}$ images were obtained using a RARE sequence (parameters: RARE factor $=8$, $\mathrm{TR} / \mathrm{TE}=1000 / 11 \mathrm{~ms}$, matrix size $=64 \times 32, \mathrm{FOV}=3 \times 1.1 \mathrm{~cm}$, slice thickness $=1 \mathrm{~mm}, \mathrm{NA}=64$, slices $=6$ ).

\section{Histology and Immunofluorescence}

Colon tissues were fixed in 2\% PFA in PBS and analyzed using histology and immunofluorescence $(n=3$ mice per group). Segments of the fixed colon tissues were embedded in paraffin, cut into $5 \mu \mathrm{m}$-thick transverse sections, and stained with hematoxylin and eosin $(\mathrm{H} \& \mathrm{E})$. The $\mathrm{H} \& \mathrm{E}$-stained slides were evaluated by an independent pathologist blinded to the groups studied. Twelve sections from different parts of the entire length of the colon were evaluated at high power magnification $(400 \times)$. Briefly, the degree of inflammation present was graded on a scale of 0 to 4 as follows: (0) No inflammatory cell infiltration, (1) few scattered inflammatory cells in lamina propria, (2) more obvious infiltrate with some extension into submucosa, (3) expansion of lamina propria with a mixed infiltrate with intraepithelial infiltration and extension to submucosa without obvious ulceration and (4) acute inflammation with ulceration.

For immunofluorescence, colon tissues from untreated $(n=3)$ and clodronate-treated $(n=2)$ groups were used. In untreated group, colon sections were stained on day 2 and day 30 after PFC administration. In clodronate-treated groups, colon sections were stained on day 2 after the first imaging session. Fixed colon tissues were frozen in liquid nitrogen and embedded in Tissue-Tek optimal cutting temperature compound (Ted Pella, Redding, CA, USA). Tissue blocks were cut into $7 \mu \mathrm{m}$-thick sections using a cryotome at $-30{ }^{\circ} \mathrm{C}$ Sections were mounted on glass slides and briefly washed with PBS and permeabilized by $0.1 \%$ Triton X-100 (Sigma-Aldridge, St Louis, MO, USA). Immunostaining was performed using primary antibodies against macrophages (F4/80), neutrophils (7/4), monocytes (Ly 6c) and endothelial cells (CD31). All primary antibodies were obtained from AbD Serotec, Raleigh, NC, USA. Secondary antibodies conjugated with Alexa 488 (AbD Serotec) were used to probe the marker expression levels. Confocal microscopy of the tissues was performed to examine the cellular colocalization of Alexa 488 with the PFC-DiI droplets using an Olympus Fluoview 1000 confocal microscope (Olympus, Center Valley, PA, USA).

\section{Nuclear Magnetic Resonance (NMR) Spectroscopy}

NMR spectroscopy of bowel samples was used to assay the total ${ }^{19} \mathrm{~F}$ content. Freshly isolated colon samples $\left.(n=2)\right)$ from the untreated group were immersed in RNAlater 
solution (Ambion, Austin, TX, USA), cut longitudinally and cleaned. RNAlater was used to preserve the sample RNA for subsequent PCR analysis. Each colon was divided into six segments, with three each in the ascending and descending colon, and each segment was weighed. Tissue segments, immersed in RNAlater, were loaded into $5 \mathrm{~mm}$ NMR tubes. The specimen size was small enough to fit entirely within the homogenous receptive field of the NMR probe so that all ${ }^{19} \mathrm{~F}$ was detected. A $5 \mu \mathrm{l}, 1 \% \mathrm{v} / \mathrm{v}$, aliquot of trifluoroacetic acid (TFA) was placed in a flame-sealed $1 \mathrm{~mm}$ diameter quartz capillary tube and positioned inside the NMR tube next to the tissue sample; this TFA reference contained a total of $N_{F}=1.2 \times 10^{18}$ fluorine atoms. A one-dimensional ${ }^{19} \mathrm{~F}$ NMR spectrum was acquired for each sample at $470 \mathrm{MHz}$ (Bruker) with 32 averages and a delay time of $5 \mathrm{~s}$. Two distinct peaks were observed at approximately $-76 \mathrm{ppm}$ for TFA and $-91.5 \mathrm{ppm}$ for the PFC. The total fluorine content $\left(C_{F}\right)$ of the sample was calculated from the integrated peak areas for the PFC $\left(I_{P F C}\right)$ and TFA $\left(I_{T F A}\right)$ reference using the formula $C_{F}=I_{P F C} N_{F} / I_{T F A}$. Results were normalized to the tissue weight, yielding the ${ }^{19} \mathrm{~F}$ atoms per gram tissue.

\section{qRT-PCR}

After NMR, quantitative PCR analysis of the same colon samples was performed to assay macrophage burden. Tissue RNA was extracted using trizol/chloroform following manufacturer's instruction (Invitrogen, Carlsbad, CA, USA). RNA recovery was measured using a Smart-Spec spectrophotometer (Bio-Rad, Hercules, CA, USA). A $5 \mu \mathrm{g}$ RNA aliquot was used for first-strand cDNA synthesis using Superscript Reverse Transcriptase (Invitrogen) with anchored primers (oligo- $\mathrm{dT}_{23}$ ) following the manufacturer's protocol. Taqman qRT-PCR probes and primers for mouse glyceraldehyde-3-phosphate dehydrogenase (GAPDH) and CD68 were designed using Vector NT (Invitrogen). Primers and probes were obtained from Integrated DNA Technologies (Coralville, IA, USA).

The primers and probe oligosequences were (1) GAPDH (forward primer: 5'-TGAACGGGAAGCTCACTGGCAT; reverse primer: $5^{\prime}$-TGCCTGCTTCACCACCTTCTTG; probe: 5'-HEX/CCGCCTGGAGAAACCTGCCAAGTA) and (2) CD68 (forward primer: 5'-ACCTACATCAGAGCCCGAGT; reverse primer: 5'-CATGAATGTCCACTGTGCTG; probe: 5'-FAM/ TTCCACCGCCATGTAGTCCAG).

For qRT-PCR, $0.25 \mu \mathrm{l}$ of cDNA templates was added to a mixture of $200 \mathrm{nM}$ forward primers, $200 \mathrm{nM}$ reverse primers, $200 \mathrm{nM}$ probes for both GAPDH and CD68 and qPCR master mix (Stratagene, La Jolla, CA, USA) according to the manufacturer's instructions. The PCR was then held at $95^{\circ} \mathrm{C}$ for $10 \mathrm{~min}$ followed by 40 cycles of $95^{\circ} \mathrm{C}$ for $30 \mathrm{~s}, 58^{\circ} \mathrm{C}$ for $60 \mathrm{~s}$ and $72^{\circ} \mathrm{C}$ for $30 \mathrm{~s}$, followed by $72^{\circ} \mathrm{C}$ for $3 \mathrm{~min}$ and cooling to $4{ }^{\circ} \mathrm{C}$ The difference between threshold cycle $(\mathrm{Ct})$ values of GAPDH and $\mathrm{CD} 68$ were used to calculate the relative expression of CD68 between different segments of the colon. Relative expression of CD68 was determined by $2^{\Delta \mathrm{Ct}}$, where $\Delta \mathrm{Ct}=\mathrm{Ct}_{\mathrm{GAPDH}}-\mathrm{Ct}_{\mathrm{CD} 68}$. Concentration of the probes, primers and cDNA required for the reaction was optimized by varying the concentrations of these constituents systematically to obtain maximal amplification. CD68 expression was normalized to GAPDH expression in the colon samples by qRT-PCR. NMR spectroscopy data and qRT-PCR data was plotted in a scattered plot and fitted with linear least square regression analysis (OriginLab, Northampton, MA, USA).

\section{RESULTS}

\section{In Vivo and In Vitro ${ }^{19} \mathrm{~F}$ MRI Reveals Inflammatory Sites in the Colon of IL-10 ${ }^{-1-}$ Mice}

Colitis in IL-10 $0^{-1-}$ mice $(n=10)$ was induced by feeding with piroxicam-doped chow for 14 days. At the end of the induction period, the $\mathrm{IL}-10^{-l-}$ mice were injected intravenously with PFC emulsion. Mice were imaged longitudinally starting from 2 days post injection. ${ }^{19} \mathrm{~F}$ MRI revealed a patchy PFC distribution in the colon wall (Figure 1a). Control C57/Bl6 mice $(n=4)$, also receiving intravenous $\mathrm{PFC}$, displayed no significant ${ }^{19} \mathrm{~F}$ signal in colon wall (Figure 1b). In all mice, ${ }^{19} \mathrm{~F}$ signal was detected in spleen and liver. Axial slices from the entire abdomen in the IL-10 ${ }^{-1-}$ mice were stacked and rendered in 3D to visualize the extent and distribution of PFC in the colon in vivo (Figure 1c). We confirmed the in vivo results using high-resolution MRI of the excised, fixed colon (Figure 1d). The appearance of the PFC distribution in the colon wall was smooth and continuous in certain bowel regions, but in other regions it appeared patchy with distinct foci (Figure 1d).

\section{Quantitative Analysis of Bowel Inflammation by ${ }^{1} \mathrm{H}$ and ${ }^{19} \mathrm{~F}$ MRI}

Bowel inflammation was assessed initially by measuring the thickness of bowel wall in $\mathrm{T}_{2}$-weighted ${ }^{1} \mathrm{H}$ anatomic scans (Figure 2a). Image analysis revealed that in ascending colon, the mean thickness of the colonic wall was $1.475 \pm 0.12 \mathrm{~mm}$ in IL- $10^{-/-}$mice compared with $0.55 \pm 0.05 \mathrm{~mm}$ in control mice $(P=0.0008, t$-test $)$. In descending colon, wall thickness was $0.53 \pm 0.1 \mathrm{~mm}$ in IL- $10^{-1-}$ mice compared with $0.39 \pm$ $0.05 \mathrm{~mm}(P=0.16, t$-test $)$ in control mice (Figure $2 \mathrm{~b})$.

The IL-10 $10^{-1-}$ mice were longitudinally imaged for 30 days following PFC emulsion administration $(n=4)$. From the image data, the PFC distribution and apparent number of ${ }^{19} \mathrm{~F}$ atoms present in the colon wall in various regions was quantified relative to a reference capillary placed along the animal's torso containing $2.34 \times 10^{17}{ }^{19} \mathrm{~F}$ atoms $/ \mathrm{mm}^{3}$ (Figure 2b). ${ }^{31}$ In the majority of mice, the PFC accumulation was higher in ascending colon compared with descending colon. The ${ }^{19} \mathrm{~F}$ signal peaked at day 9 after injection, and by day 30 the signal decreased to the level observed on day 2. In control C57/Bl6 mice, no ${ }^{19} \mathrm{~F}$ signal was observed in the colon in any of the imaging sessions. There was strong ${ }^{19} \mathrm{~F}$ signal found in the liver and spleen. 

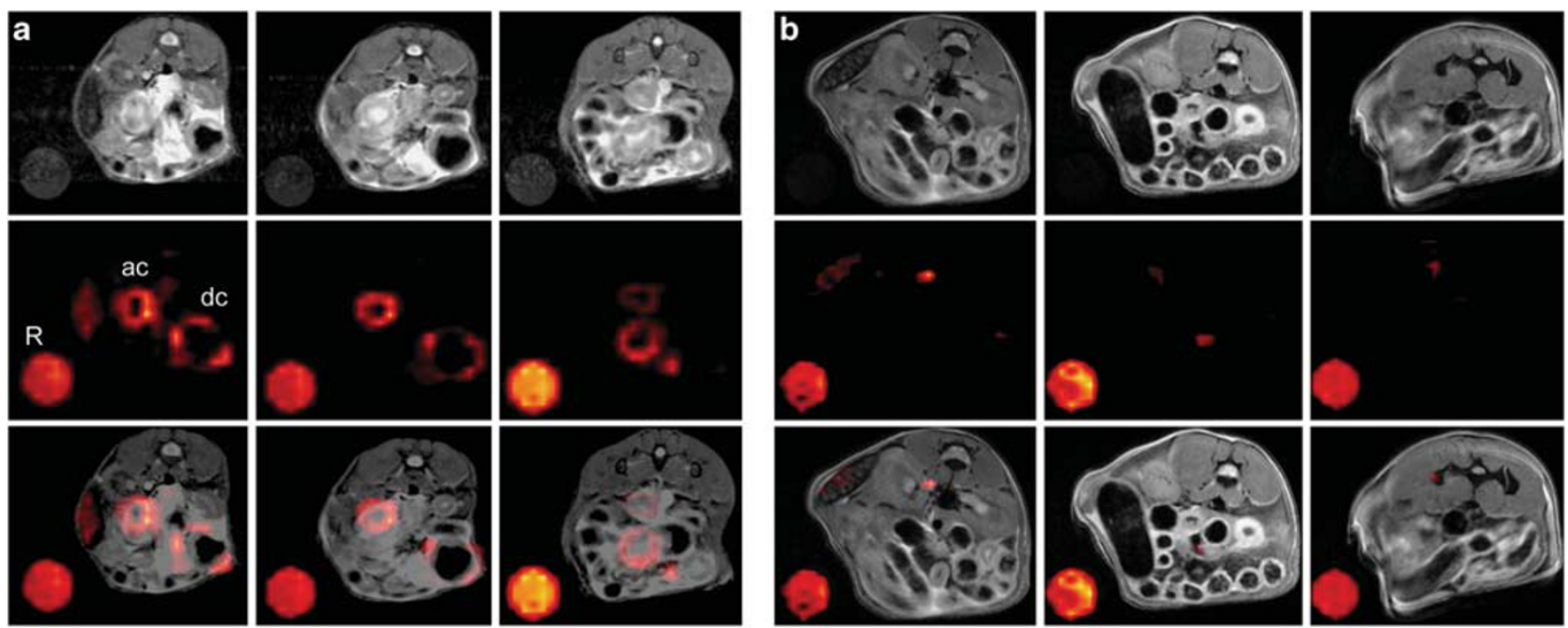

C

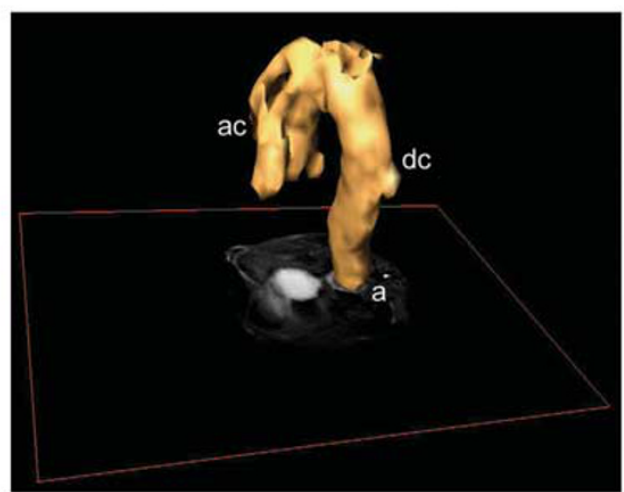

d
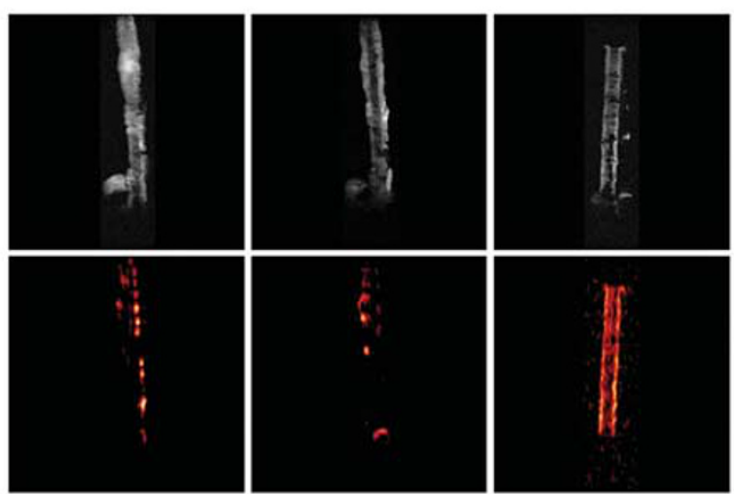

Figure $1{ }^{1} \mathrm{H} /{ }^{19} \mathrm{~F}$ magnetic resonance imaging (MRI) reveals perfluorocarbon (PFC) distribution in colon walls in a representative inflammatory bowel disease mouse. (a) In vivo axial slices through the abdomen of a single mouse shows PFC accumulation in the ascending (ac) and descending colon (dc) in a interleukin-10-1- $\left(\mathrm{IL}-10^{-1-}\right)$ mouse 2 days post injection. Upper panel shows ${ }^{1} \mathrm{H}$ images (grayscale), middle panel shows corresponding ${ }^{19} \mathrm{~F}$ images (pseudocolor) and lower panel shows composite ${ }^{1} \mathrm{H} /{ }^{19} \mathrm{~F}$ images. ${ }^{19} \mathrm{~F}$ signals are also observed in the spleen and possibly lymph nodes. ' $\mathrm{R}$ ' represents a reference tube alongside the torso containing PFC emulsion. Each image slice was scaled separately for optimal visualization. (b) Representative axial slices through the abdomen of control C57/BL6 mice shows no ${ }^{19} \mathrm{~F}$ signal in the colon. ${ }^{19} \mathrm{~F}$ signal is observed in the spleen. (c) $3 \mathrm{D}$ rendering of the in vivo $19 \mathrm{~F}$ MRI data from the abdomen in the IL-10 $10^{-1-}$ mice; substantial inflammation in the ascending and descending colon is apparent. Here, 'ac' $=$ ascending colon, ' $\mathrm{dc}$ ' $=$ descending colon and ' $\mathrm{a}$ ' $=$ anus. (d) High-resolution ex vivo MRI shows the ${ }^{1} \mathrm{H}$ anatomy (upper panels) and distribution of ${ }^{19} \mathrm{~F}$ signal (lower panels) in colon walls in a single $\mathrm{IL}-10^{-1-}$ mouse.
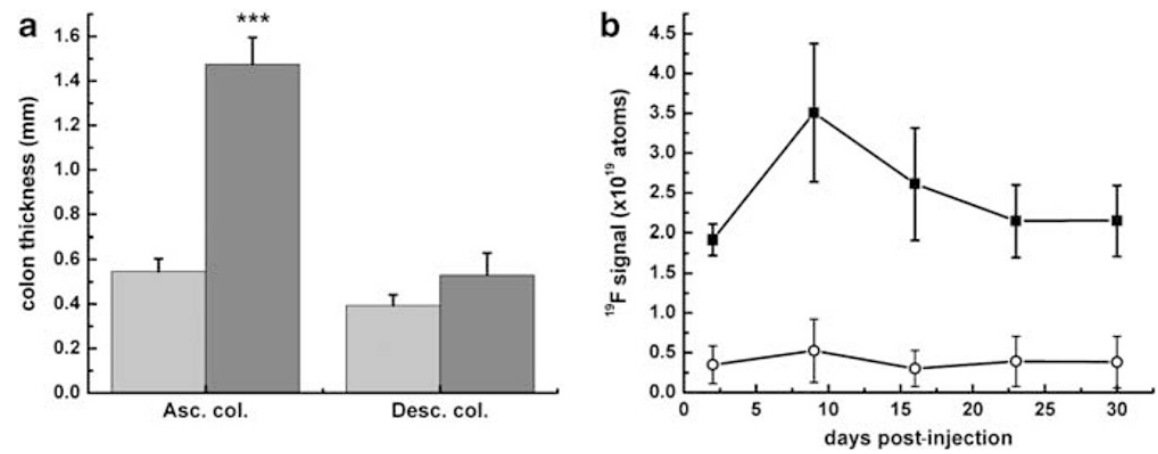

Figure 2 Quantitative analysis of colon inflammation using magnetic resonance imaging (MRI) data. (a) Colon thickness was measured from $\mathrm{T}_{2}$-weighted ${ }^{1} \mathrm{H}$ MRI data on day 2 post injection. Colon thickness in ascending colon $(P=0.0008)$, and descending colon $(P=0.16)$ in interleukin-10 ${ }^{-1-}\left(\mathrm{IL}-10^{-1-}\right)$ mice (dark gray) is higher than in control animals (light gray). (b) Longitudinal analysis of ${ }^{19} \mathrm{~F}$ signal in colon walls in IL-10 $0^{-1-}$ mice measured over 30 days. The ${ }^{19} \mathrm{~F}$ signal in the ascending colon (square) was higher than descending colon (circle) on all days. Peak signal intensity was observed on day 9 in ascending colon, and by day 30 the signal decreased to the level observed on day 2 . Control C57/Bl6 mice did not show any ${ }^{19} \mathrm{~F}$ signal in the colon (data not shown). Statistical analyses were performed using one-tailed $t$-test. 


\section{Histology of Colon Reveals Mucosal Hyperplasia and Infiltration of Mononuclear Cells}

At the end of induction period, mice were euthanized and colon tissues were excised. Average weight of ascending colon $(P=0.004, t$-test $)$ and descending colon $(P=0.001, t$-test $)$ in IL- $10^{-1-}$ mice was significantly higher than that of the colon in control mice (Figure 3a). Histological sections stained with $\mathrm{H} \& \mathrm{E}$ revealed inflammation in the bowel wall characterized by mucosal hyperplasia, goblet cell depletion and infiltration of inflammatory mononuclear cells (Figure $3 \mathrm{~b}$ ). Lymphocytes and macrophages were the predominant infiltrates. In a blind histopathological analysis, the average colitis score in the IL- $10^{-/-}$mice was significantly higher compared with control mice (Figure 3c, $P=0.012, t$-test). Colon inflammation was predominantly observed in ascending colon.

\section{Macrophage Burden Forms the Basis of the ${ }^{19} \mathrm{~F}$ MRI Signals in the Bowel}

We characterized the cellular basis of the ${ }^{19} \mathrm{~F}$ signal in the colon by confocal immunofluorescence microscopy. Prior to injection, the PFC emulsion was pre-labeled with a DiI fluorophore. ${ }^{20}$ DiI (red), adherent to the PFC droplets, was present exclusively within F4/80 macrophages in the colon from day 2 to day 30 (green, Figures $4 \mathrm{a}$ and b). Approximately $15-20 \%$ of the macrophages in the colonic wall contained PFC droplets. The correlation coefficient between F4/80-positive macrophages and observed PFC droplets is 0.99. The PFC droplets did not colocalize with neutrophils, endothelial cells $(\mathrm{CD} 31)$ or ly6 $\mathrm{C}^{\text {hi }}$ cells (Figures $4 \mathrm{c}$ and d). These data suggest that ${ }^{19} \mathrm{~F}$ MRI exclusively measures macrophage content in the colon, and quantification of the apparent ${ }^{19} \mathrm{~F}$ signal reflects macrophage burden.

We further characterized the basis of ${ }^{19} \mathrm{~F}$ signal in the bowel by directly correlating the level of macrophage mRNA (CD68) expression with the total ${ }^{19} \mathrm{~F}$ signal in colon segments. Excised colon was divided into six segments. The total ${ }^{19} \mathrm{~F}$ signal in a colon segment was quantified by ${ }^{19} \mathrm{~F}$ NMR spectroscopy and normalized to the weight of each segment.
The macrophage load in the segment was quantified by qRTPCR analysis for the expression of macrophage marker CD68 normalized to the expression of the house-keeping gene GAPDH. The ${ }^{19} \mathrm{~F}$ signal per tissue weight showed a linear correlation with normalized CD68 expression in the diseased colon (Figure $4 \mathrm{e}, R=0.65, P=0.03$ ).

\section{Selective Ablation of Macrophages In Vivo Attenuates ${ }^{19} \mathrm{~F}$ Signal in the Colon}

To further test whether macrophages are responsible for ${ }^{19} \mathrm{~F}$ signal in the bowel, selective ablation of macrophages was performed. Mice were prophylactically treated with clodronate liposomes during the colitis induction phase. Intraperitoneal treatment of clodronate liposomes ablates circulating monocytes in the blood and resident macrophages in the abdomen. ${ }^{29}$ We observed that clodronate liposome treatment significantly reduced ${ }^{19} \mathrm{~F}$ signal in ascending $(P=0.001, t$-test $)$ and descending colon $(P=0.05 t$-test $)$ compared with the untreated mice (Figure $5 \mathrm{a}, n=3$ ). Histological analysis showed significant decrease in the colitis score in the ascending colon $(P=0.013, t$-test $)$ after clodronate liposome treatment. The decrease in colitis score was less apparent in the descending colon $(P=0.06, t$-test $)$, probably due to the low initial inflammatory response in the descending colon (Figure 5b, $n=3$ ). In addition, immunofluorescence revealed a decrease in macrophage density in the colon, and there was no apparent accumulation of PFC-DiI in the macrophages (Figure $5 c$ ).

\section{Impact of Dexamethasone and CsA on the Development of Colitis in IL-10 ${ }^{-1-}$ Mice}

We tested the prophylactic effects of commonly used clinical drugs on the induction of colitis in IL- $10^{-1-}$ mice, with the goal of evaluating whether PFC and ${ }^{19} \mathrm{~F}$ MRI could detect putative therapeutic effects in vivo. Prophylactic treatment with dexamethasone $(n=5)$ showed a significant increase in the accumulation of PFC in the colon, especially in the
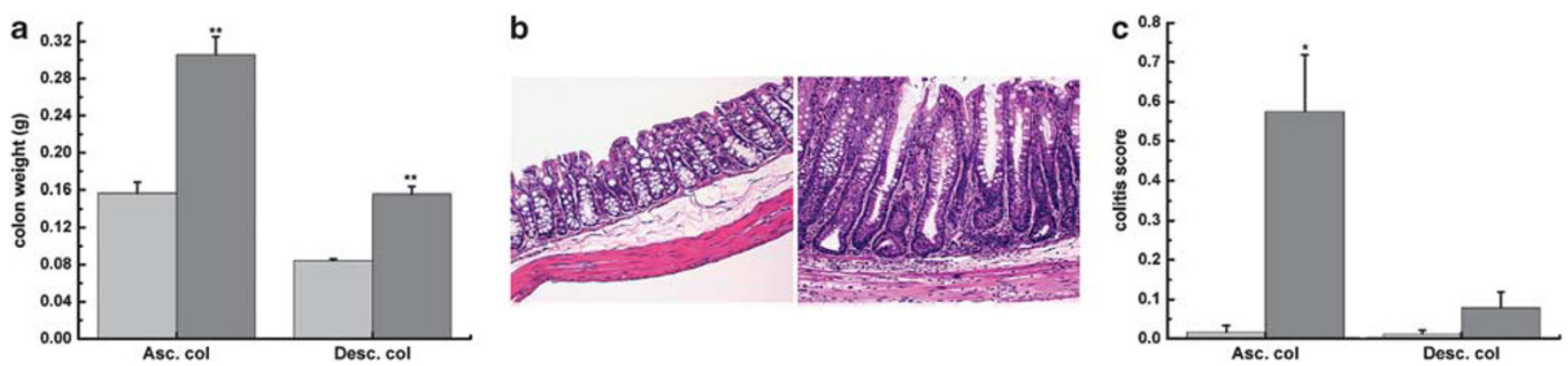

Figure 3 Gross and microscopic examination of excised colon reveals inflammation. (a) Average weight of ascending colon $(P=0.004)$ and descending colon $(P=0.001)$ in interleukin-10 $10^{-1-}\left(\mathrm{IL}-10^{-1-}\right)$ mice was significantly higher than that of the colon in control mice. (b) Representative H\&E (hematoxylin and eosin)-stained sections from the descending colon show normal colon (left panel) and inflamed colon (right panel) from IL-10 ${ }^{-1-}$ mouse. Inflammation is characterized by mucosal hyperplasia, goblet cell depletion and mononuclear cell infiltration (magnification $200 \times$ ). (c) Average colitis score is significantly higher in IL-10 $0^{-/-}$mice (dark gray bars) compared with the controls (light gray bars). Colitis is observed predominantly in the ascending colon in the IL-10 ${ }^{-1-}$ mice $(P=0.012)$. Statistical analyses were performed using one-tailed $t$-test. 

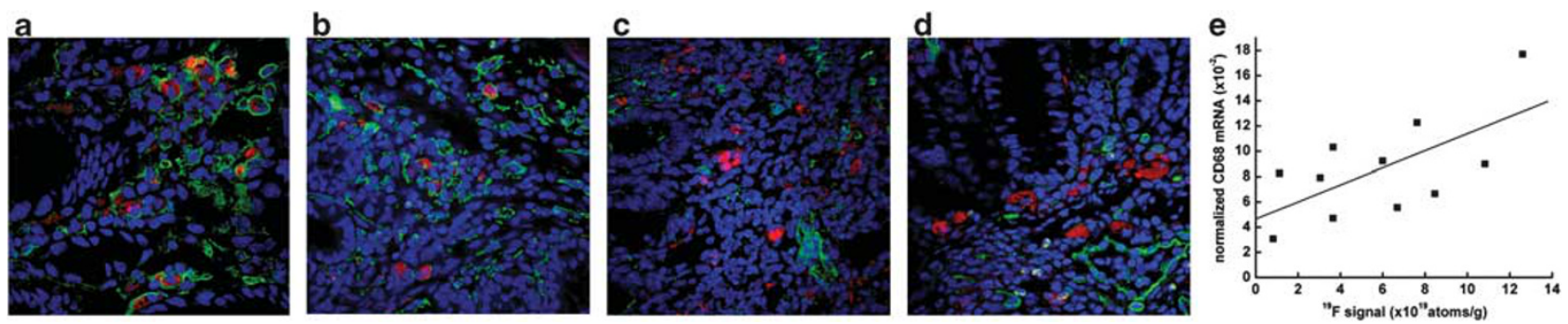

Figure 4 Macrophage burden forms the basis of ${ }^{19} \mathrm{~F}$ signal in the colon. (a) Immunohistochemistry of the colon in interleukin-10 ${ }^{-/-}\left(\right.$IL- $10^{-/-}$) mice shows that perfluorocarbon (PFC)-Dil (red) is localized within the macrophages (F4/80, green) at 2 days and (b) 30 days after PFC injection. Nuclei are stained with Hoechst 33342 (blue). Overall, there is minimal amount of PFC-Dil that is not colocalized with the F4/80. (c) Shows that there is no colocalization with ly6cpositive cells (green) and PFC-Dil. (d) Shows that there is no colocalization with endothelial cells (CD31, green) and PFC-Dil (magnification $630 \times$ ).

Representative sections are from the descending colon. (e) Shows a positive correlation between ${ }^{19} \mathrm{~F}$ signal in the colon segments and macrophage mRNA in the same segments of the colon $\left(P=0.03, R^{2}=0.65\right)$. Macrophage mRNA expression is normalized to glyceraldehyde-3-phosphate dehydrogenase (GAPDH) expression levels, and ${ }^{19} \mathrm{~F}$ nuclear magnetic resonance signal is normalized by tissue weight.
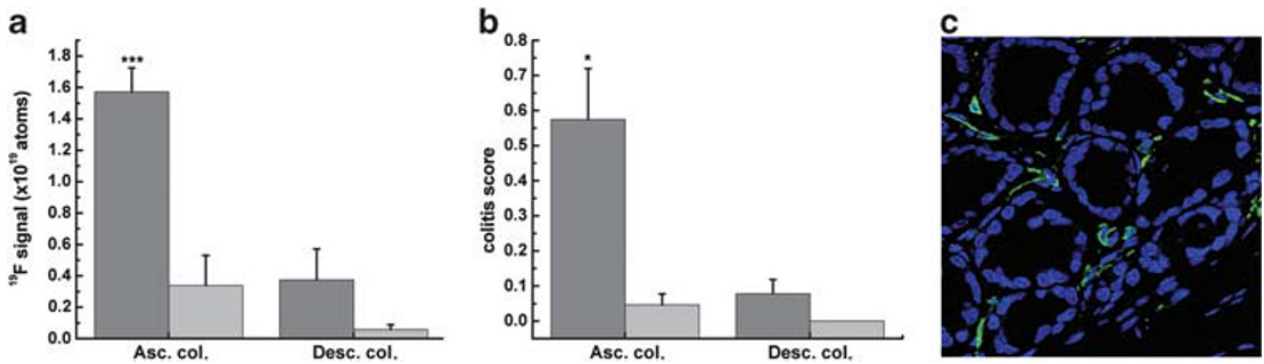

Figure 5 Ablation of macrophages in vivo attenuates ${ }^{19} \mathrm{~F}$ signal in the colon. (a) Macrophages in circulation were ablated by treating with clodronate liposomes. Treating animals (light gray) during the induction phase of colitis reduced ${ }^{19} \mathrm{~F}$ signal in both ascending $(P=0.001)$ and descending colon $(P=0.05)$ compared with the untreated mice (dark gray). (b) Histological analysis showed that colitis score is reduced in ascending colon $(P=0.013, n=3)$

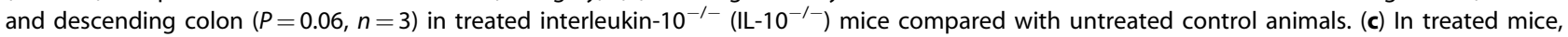
immunohistochemistry displayed sparse macrophages in the colon (green), and no perfluorocarbon (PFC)-Dil was observed in the colon sections. The representative section is from the descending colon. Statistical analyses were performed using one-tailed $t$-test.
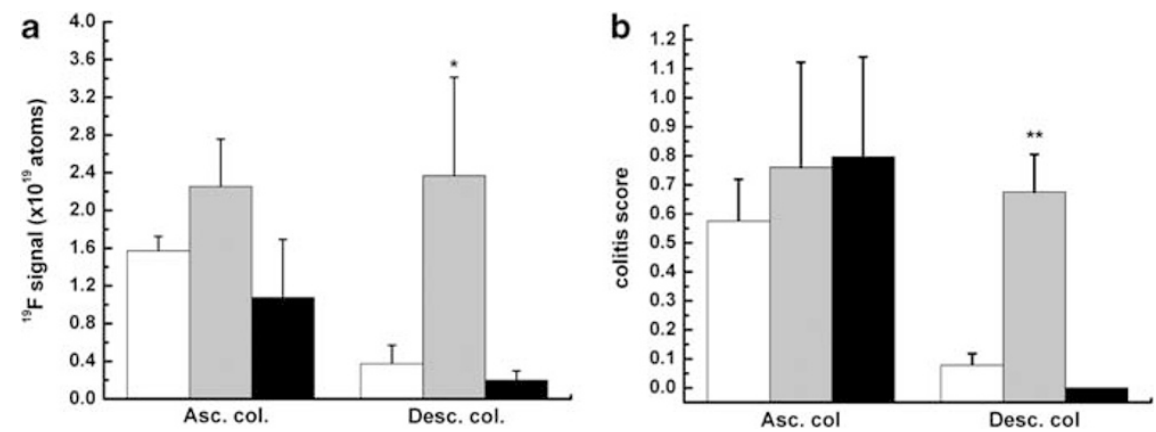

Figure 6 Impact of dexamethasone and cyclosporine A (CSA) on the development of inflammatory bowel disease. (a) Prophylactic treatments with dexamethasone increased ${ }^{19} \mathrm{~F}$ signals in the colon, especially in the descending colon $(P=0.048)$. CsA (black) show minimal effect ${ }^{19} \mathrm{~F}$ signals $(P=0.1)$ compared with untreated (white). In the ascending colon, both dexamethasone (gray, $P=0.1)$ and $\mathrm{CsA}(P=0.2)$ did not exhibit any therapeutic effects. (b) Colitis score in the descending colon in dexamethasone-treated mice was significantly increased $(P=0.005)$, and colitis score in CsA-treated mice $(P=0.06)$ was decreased. There was no significant change in colitis score in the ascending colon for dexamethasone- $(P=0.3)$ and $\operatorname{CsA}$-treated $(P=0.3)$ mice compared with untreated controls. Statistical analyses were performed using one-tailed $t$-test.

descending colon $(P=0.048 t$-test $)$, suggesting that dexamethasone does not prevent the development of colitis at the dose tested in these mice (Figure 6a). Prophylactic treatment with CsA $(n=4)$ showed a trend towards reduced
PFC accumulation in the descending colon, but the effect was not statistically significant (Figure 6a, $P=0.1 t$-test). In the ascending colon, both dexamethasone $(P=0.1, t$-test $)$ and CsA $(P=0.2, t$-test $)$ did not exhibit any therapeutic effects. 
Histological analysis showed an increase in colitis score in dexamethasone-treated mice (Figure $6 \mathrm{~b}, P=0.005, t$-test) and a decrease in colitis score in CsA-treated mice in the descending colon (Figure $6 \mathrm{~b}, P=0.06, t$-test). In ascending colon, there was no significant change in colitis score in both dexamethasone $(P=0.3$, $t$-test $)$ and CsA-treated $(P=0.3$, $t$-test) mice compared with untreated controls.

\section{DISCUSSION}

In this paper, we show that macrophage activity associated with inflammation in the colon of IL- $10^{-1-}$ mice can be non-invasively detected and scored using ${ }^{19} \mathrm{~F}$ MRI utilizing a novel PFC tracer-imaging agent. Using immunofluorescence, qRT-PCR and in situ macrophage ablation, we demonstrate that PFC is localized within macrophages and that the cell quantity is reflected in the magnitude of the measured ${ }^{19} \mathrm{~F}$ signal in the colon. Furthermore, we show that the method is suitable to assay the effects of putative therapeutics used to treat colitis. The total imaging time for ${ }^{19} \mathrm{~F}$ MRI used in this work was $\sim 8 \mathrm{~min}$, which is practical for routine preclinical studies and testing of therapeutic test articles.

The non-invasive nature of ${ }^{19} \mathrm{~F}$ MRI provides rapid assessment of the extent and 3D distribution of bowel inflammation in a manner that is not feasible by invasive procedures like biopsy or necropsy. Importantly, the extent of colon inflammation and age of onset are key factors that determine the progression of colitis into colitis-associated colon cancer. ${ }^{1}$ Because there is no endogenous MRI-detectable fluorine in the bowel, the only source of ${ }^{19} \mathrm{~F}$ signal is inflammation-associated macrophage activity, and thus detection is unambiguous. The absence of background ${ }^{19} \mathrm{~F}$ enables reliable lesion quantification, which is often challenging with various ${ }^{1} \mathrm{H}$ MRI modalities. In our study, it was difficult to assess inflammation by measuring bowel thickness by ${ }^{1} \mathrm{H}$ MRI alone, especially in descending colon where inflammation was mild. The ${ }^{19} \mathrm{~F}$ signals were readily detected in the descending colon even when the inflammation was mild. Moreover, 3D reconstructions of bowel lesions are feasible without manual segmentation, offering views of the colon within the context of the whole abdominal cavity that provide an accurate picture of the extent and distribution of the diseased regions.

As a class of molecules, many PFCs are considered safe for in vivo applications. Several PFCs have been used in human clinical trials as artificial oxygen carriers. ${ }^{32}$ The blood half-life of the PFC used in this study is $\sim 12 \mathrm{~h}$ in mice. In tissues, PFC accumulates in the reticuloendothelial system initially and is slowly cleared via the lung. Recently, numerous studies have investigated the impact of PFC cell labeling on cellular phenotype and function in primary immune cells using a variety of sensitive in vitro assays, eg, in the context of human dendritic cells $\mathrm{s}^{30,33}$ and murine $\mathrm{T}$ cells. ${ }^{31,34,35}$ Cytotoxicity following PFC labeling was minimal, and there were no phenotypic alterations to the cells after labeling. These studies and others ${ }^{34,36-38}$ label cells with various PFC molecules in vitro with the goal of performing in vivo ${ }^{19} \mathrm{~F}$ celltracking studies after cell transfer to a subject, eg, to monitor T-cell migration in experimental models of diseases. In a prior study using PFC-labeled antigen-specific $\mathrm{T}$ cells, we reported extra-intestinal loci of inflammation in pancreas in a mucin $1^{+}\left(\mathrm{MUC1}^{+}\right)$IL-10 $0^{-/-}$transgenic mouse model of IBD using ${ }^{19} \mathrm{~F}$ NMR and MRI. ${ }^{34}$ We note that in the IL-10 ${ }^{-1-}$ model utilized in this study there was no evidence of pancreatitis.

Quantitatively, in vitro PFC emulsion uptake by phagocytic cells, such as fetal skin-derived dendritic cells, is empirically found to be on the order of $\sim 5 \times 10^{12}$ fluorine atoms per cell; ${ }^{30}$ for non-phagocytic cells, such as 91 glioma cells and $\mathrm{T}$ cells, uptake ranges from approximately $2 \times 10^{11}$ to $1 \times 10^{12}$ fluorine atoms per cell. ${ }^{34,37}$ Assuming that macrophages uptake $\sim 5 \times 10^{12}$ atoms per cell in vivo, we estimate that the magnitude of macrophages in the bowel wall is of order $\sim 3 \times 10^{6}$ cells based on our measured ${ }^{19} \mathrm{~F}$ levels in vivo (Figure 2a). We note that the mean PFC uptake per macrophage in vivo is not known and difficult to measure, unlike the ex vivo labeling experiments. From immunofluorescence, it is estimated that $15-20 \%$ of macrophages in the colon contained PFC-DiI.

We demonstrated that ablation of macrophages in the circulation and abdominal organs with clodronate liposomes greatly reduces ${ }^{19} \mathrm{~F}$ signal in the colon. This result further demonstrates the selective labeling of macrophages by PFCs in vivo. Histological scoring demonstrated that macrophage ablation reduced inflammation in colon. This result indicates the importance of macrophages in the development of IBD.

We tested the prophylactic effects of two drugs, including dexamethasone and CsA, in IBD induction; the goal of these tests was to determine if ${ }^{19} \mathrm{~F}$ MRI could be used to detect any putative therapeutic effects. Both drugs are used clinically to treat patients with IBD. ${ }^{39}$ In our model, dexamethasone increased inflammation, especially in the descending colon, whereas CsA had a small effect on the induction of colitis. We note that facilitation of colitis with dexamethasone was also observed in the acute phase of DSS-induced colitis, ${ }^{40,41}$ but it ameliorated colitis in the chronic phase of DSS-induced colitis. ${ }^{41}$ The exact mechanisms of these effects are currently not known. Differential effects of corticosteroids on the immune cells could partly explain the observed phenomenon. ${ }^{42}$ Neutrophils, the mediators of acute inflammation, are relatively resistant to corticosteroid action and may promote the initial acute inflammation phase in piroxicam-induced intestinal damage in our model which in turn could trigger uncontrolled inflammation in an immune-deficient model such as IL- $10^{-1-}$ mice. Dexamethasone is also known to aggravate the adverse gastrointestinal effects, such as perforation and bleeding, when combined with non-steroidal, anti-inflammatory drugs such as piroxicam. ${ }^{43} \mathrm{CsA}$, on the other hand, reduced the severity of inflammation in the acute phase of DSS-mediated colitis by upregulating transforming 
growth factor- $\beta^{44}$ in the intestine. CsA reduces inflammation by suppressing the secretion of IL-2 by activated T cells. ${ }^{45}$ Activated $\mathrm{CD} 4{ }^{+} \mathrm{T}$ cells are the primary effectors that perpetuate colitis in IL-10 $0^{-/-}$mice that act synergistically with macrophages to promote inflammation. The initial induction phase of piroxicam-induced colitis in IL- $10^{-/-}$mice may be similar to acute phase of DSS-induced colitis.

In this study, we have employed a prophylactic IBD treatment model. Although, prophylactic treatment models can provide important insights into the mechanisms of disease, and in our study the basis of the ${ }^{19} \mathrm{~F}$ MRI signal generation, we acknowledge that it is less relevant clinically. In this work, our attempt was to establish a quantitative method of measuring bowel inflammation, and therapeutic interventions were primarily used for that purpose.

The MRI-active PFC molecule (perfluoropolyether) used in this study, designed for optimal MRI sensitivity (ie, large number of NMR-equivalent ${ }^{19} \mathrm{~F}$ 's and short $\mathrm{T}_{1} / \mathrm{T}_{2}$ ratio), has not yet been approved for clinical use. Related PFC molecules, such as perfluorooctlybromide, have been used as oxygen transport reagents in human clinical trials and have been used in MRI studies. ${ }^{46,47}$ However, perfluorooctylbromide is non-ideal for MRI, as it has a long $\mathrm{T}_{1}$ and displays multiple ${ }^{19} \mathrm{~F}$ NMR peaks, which significantly compromises sensitivity as only one single peak can be used for imaging, and the other peaks must be suppressed using pre-excitation saturation pulses. Our view is that the results presented herein help provide a rationale for pursuing the development of optimal clinical MRI diagnostic reagents for IBD that are based on PFC emulsions. Moreover, the feasibility of performing ${ }^{19} \mathrm{~F}$ MRI scans on a clinical scanner has been demonstrated in many studies ${ }^{38,48}$ with the addition of a dualtuned ${ }^{19} \mathrm{~F} /{ }^{1} \mathrm{H}$ radio-frequency coil.

Imaging using ${ }^{18} \mathrm{FDG} \mathrm{PET}^{49}$ and endomicroscopic-imaging approaches ${ }^{50}$ have also been used for quantifying bowel inflammation. ${ }^{18} \mathrm{FDG}$ imaging has been used in human studies. ${ }^{19} \mathrm{~F}$ MRI may be a promising alternative to both of these techniques. ${ }^{18}$ FDG PET measures metabolic activity and our approach measures macrophage content in the inflammatory sites. Both of these approaches are capable of measuring inflammatory activity. PET is sensitive but involves radioactive tracers, can have high background and lower spatial resolution. Moreover, it requires CT for anatomical localization. ${ }^{19} \mathrm{~F}$ MRI does not use ionizing radiation, has better spatial resolution and ${ }^{1} \mathrm{H}$ and ${ }^{19} \mathrm{~F}$ MRI can be performed on the same instrument to obtain complementary information. Endoscopic approaches are invasive, whereas ${ }^{19} \mathrm{~F}$ MRI is capable of 3D visualization of bowel inflammation non-invasively.

Overall, macrophages have an important role in the pathogenesis of a large number of chronic inflammatory diseases and cancers. ${ }^{19} \mathrm{~F}$ MRI is capable of in vivo visualization and quantification of macrophage activity. We believe that in the future, ${ }^{19} \mathrm{~F}$ MRI technology will be an effective tool for monitoring macrophage activities in disease and for assessing treatment response.

\section{ACKNOWLEDGEMENTS}

We thank Nico van Rooijen for kindly providing the clodronate liposomes and Kevin Hitchens for critical reading of the manuscript. We acknowledge support from the National Institutes of Health grant R01-CA134633 and the Pittsburgh NMR Center for Biomedical Research, supported by P41-EB001977.

\section{DISCLOSURE/CONFLICT OF INTEREST}

ETA is a consultant for Celsense.

1. Winawer SJ, Fletcher $\mathrm{RH}$, Miller $\mathrm{L}$, et al. Colorectal cancer screening: clinical guidelines and rationale. Gastroenterology 1997;112:594-642.

2. Schreyer AG, Seitz J, Feuerbach $S$, et al. Modern imaging using computer tomography and magnetic resonance imaging for inflammatory bowel disease (IBD). Inflamm Bowel Dis 2004;10:45-54.

3. Dijkstra J, Reeders JW, Tytgat GN. Idiopathic inflammatory bowel disease: endoscopic-radiologic correlation. Radiology 1995;197: 369-375.

4. Sheikh SZ, Plevy SE. The role of the macrophage in sentinel responses in intestinal immunity. Curr Opin Gastroenterol 2010;26:578-582.

5. Hanauer SB. Inflammatory bowel disease: epidemiology, pathogenesis, and therapeutic opportunities. Inflamm Bowel Dis 2006; 12(Suppl 1):S3-S9.

6. Mahida YR. The key role of macrophages in the immunopathogenesis of inflammatory bowel disease. Inflamm Bowel Dis 2000;6:21-33.

7. Xavier RJ, Podolsky DK. Unravelling the pathogenesis of inflammatory bowel disease. Nature 2007;448:427-434.

8. Reinecker $\mathrm{HC}$, Steffen $M$, Witthoeft $T$, et al. Enhanced secretion of tumour necrosis factor-alpha, IL- 6 , and IL- 1 beta by isolated lamina propria mononuclear cells from patients with ulcerative colitis and Crohn's disease. Clin Exp Immunol 1993;94:174-181.

9. Fujino $S$, Andoh A, Bamba $S$, et al. Increased expression of interleukin 17 in inflammatory bowel disease. Gut 2003;52:65-70.

10. Sartor RB. Pathogenesis and immune mechanisms of chronic inflammatory bowel diseases. Am J Gastroenterol 1997;92(12 Suppl):5S-11S.

11. Berg DJ, Davidson N, Kuhn R, et al. Enterocolitis and colon cancer in interleukin-10-deficient mice are associated with aberrant cytokine production and CD4(+) TH1-like responses. J Clin Invest 1996;98:10101020.

12. Neurath MF, Fuss I, Pasparakis M, et al. Predominant pathogenic role of tumor necrosis factor in experimental colitis in mice. Eur J Immunol 1997;27:1743-1750.

13. Sempere GA, Martinez Sanjuan V, Medina Chulia E, et al. MRI evaluation of inflammatory activity in Crohn's disease. Am J Roentgenol 2005;184:1829-1835.

14. Modo $M$, Hoehn $M$, Bulte JW. Cellular MR imaging. Mol Imaging 2005;4:143-164.

15. Hitchens TK, Ye Q, Eytan DF, et al. 19F MRI detection of acute allograft rejection with in vivo perfluorocarbon labeling of immune cells. Magn Reson Med 2011;65:1144-1153.

16. Ebner B, Behm P, Jacoby $C$, et al. Early assessment of pulmonary inflammation by 19F MRI in vivo. Circ Cardiovasc Imaging 2010; 3:202-210.

17. Noth U, Morrissey SP, Deichmann R, et al. Perfluoro-15-crown-5-ether labelled macrophages in adoptive transfer experimental allergic encephalomyelitis. Artif Cells Blood Substit Immobil Biotechnol 1997;25:243-254.

18. Flogel $\mathrm{U}$, Ding $\mathrm{Z}$, Hardung $\mathrm{H}$, et al. In vivo monitoring of inflammation after cardiac and cerebral ischemia by fluorine magnetic resonance imaging. Circulation 2008;118:140-148.

19. Flogel U, Su S, Kreideweiss I, et al. Noninvasive detection of graft rejection by in vivo (19) F MRI in the early stage. Am J Transplant 2011;11:235-244.

20. Ahrens ET, Young $W B, X u H$, et al. Rapid quantification of inflammation in tissue samples using perfluorocarbon emulsion and fluorine-19 nuclear magnetic resonance. Biotechniques 2011; 50:229-234.

21. Kuhn R, Lohler J, Rennick D, et al. Interleukin-10-deficient mice develop chronic enterocolitis. Cell 1993;75:263-274. 
22. Blumberg RS, Saubermann LJ, Strober W. Animal models of mucosal inflammation and their relation to human inflammatory bowel disease. Curr Opin Immunol 1999;11:648-656.

23. Schreiber $\mathrm{S}$, Heinig $\mathrm{T}$, Thiele $\mathrm{HG}$, et al. Immunoregulatory role of interleukin 10 in patients with inflammatory bowel disease. Gastroenterology 1995;108:1434-1444.

24. Tedde A, Laura Putignano A, Bagnoli S, et al. Interleukin-10 promoter polymorphisms influence susceptibility to ulcerative colitis in a gender-specific manner. Scand J Gastroenterol 2008;43:712-718.

25. Franke $A$, Balschun $T$, Karlsen $T H$, et al. Sequence variants in IL10, ARPC2 and multiple other loci contribute to ulcerative colitis susceptibility. Nat Genet 2008;40:1319-1323.

26. Weinstock JV, Blum A, Metwali A, et al. Substance $P$ regulates Th1-type colitis in IL-10 knockout mice. J Immunol 2003;171:3762-3767.

27. Watanabe $\mathrm{N}$, Ikuta K, Okazaki K, et al. Elimination of local macrophages in intestine prevents chronic colitis in interleukin-10-deficient mice. Dig Dis Sci 2003;48:408-414.

28. Ohkawara T, Nishihira J, Takeda $\mathrm{H}$, et al. Amelioration of dextran sulfate sodium-induced colitis by anti-macrophage migration inhibitory factor antibody in mice. Gastroenterology 2002;123:256-270.

29. Van Rooijen N, Sanders A. Liposome mediated depletion of macrophages: mechanism of action, preparation of liposomes and applications. J Immunol Methods 1994;174:83-93.

30. Ahrens ET, Flores $\mathrm{R}, \mathrm{Xu} \mathrm{H}$, et al. In vivo imaging platform for tracking immunotherapeutic cells. Nat Biotechnol 2005;23:983-987.

31. Srinivas M, Morel PA, Ernst LA, et al. Fluorine-19 MRI for visualization and quantification of cell migration in a diabetes model. Magn Reson Med 2007;58:725-734.

32. Kim HW, Greenburg AG. Artificial oxygen carriers as red blood cell substitutes: a selected review and current status. Artif Organs 2004;28:813-828.

33. Helfer BM, Balducci $A$, Nelson $A D$, et al. Functional assessment of human dendritic cells labeled for in vivo (19)F magnetic resonance imaging cell tracking. Cytotherapy 2010;12:238-250.

34. Kadayakkara DK, Beatty PL, Turner MS, et al. Inflammation driven by overexpression of the hypoglycosylated abnormal mucin 1 (MUC1) links inflammatory bowel disease and pancreatitis. Pancreas 2010;39:510-515.

35. Janjic JM, Srinivas M, Kadayakkara DK, et al. Self-delivering nanoemulsions for dual fluorine-19 MRI and fluorescence detection. J Am Chem Soc 2008;130:2832-2841.
36. Ruiz-Cabello J, Walczak P, Kedziorek DA, et al. In vivo 'hot spot' MR imaging of neural stem cells using fluorinated nanoparticles. Magn Reson Med 2008:60:1506-1511.

37. Kadayakkara DK, Janjic JM, Pusateri LK, et al. In vivo observation of intracellular oximetry in perfluorocarbon-labeled glioma cells and chemotherapeutic response in the CNS using fluorine-19 MRI. Magn Reson Med 2010;64:1252-1259.

38. Partlow KC, Chen J, Brant JA, et al. 19F magnetic resonance imaging for stem/progenitor cell tracking with multiple unique perfluorocarbon nanobeacons. FASEB J 2007;21:1647-1654.

39. Sands BE. Therapy of inflammatory bowel disease. Gastroenterology 2000;118(2 Suppl 1):S68-S82.

40. van Meeteren ME, Meijssen MA, Zijlstra FJ. The effect of dexamethasone treatment on murine colitis. Scand J Gastroenterol 2000;35:517-521

41. Kojouharoff G, Hans W, Obermeier F, et al. Neutralization of tumour necrosis factor (TNF) but not of IL-1 reduces inflammation in chronic dextran sulphate sodium-induced colitis in mice. Clin Exp Immunol 1997;107:353-358.

42. Parrillo JE, Fauci AS. Mechanisms of glucocorticoid action on immune processes. Annu Rev Pharmacol Toxicol 1979;19:179-201.

43. Gordon PV, Attridge JT. Understanding clinical literature relevant to spontaneous intestinal perforations. Am J Perinatol 2009;26:309-316.

44. Satoh $Y$, Ishiguro $Y$, Sakuraba $H$, et al. Cyclosporine regulates intestinal epithelial apoptosis via TGF-beta-related signaling. Am J Physio Gastrointest Liver Physiol 2009;297:G514-G519.

45. Schreiber SL, Crabtree GR. The mechanism of action of cyclosporin A and FK506. Immunol Today 1992;13:136-142.

46. Laukemper-Ostendorf S, Scholz A, Burger K, et al. 19F-MRI of perflubron for measurement of oxygen partial pressure in porcine lungs during partial liquid ventilation. Magn Reson Med 2002;47:82-89.

47. Ratner AV, Hurd R, Muller $\mathrm{HH}$, et al. $19 \mathrm{~F}$ magnetic resonance imaging of the reticuloendothelial system. Magn Reson Med 1987:5:548-554.

48. Neubauer AM, Caruthers SD, Hockett FD, et al. Fluorine cardiovascular magnetic resonance angiography in vivo at $1.5 \mathrm{~T}$ with perfluorocarbon nanoparticle contrast agents. J Cardiovasc Magn Reson 2007;9:565-573.

49. Meisner RS, Spier BJ, Einarsson S, et al. Pilot study using PET/CT as a novel, noninvasive assessment of disease activity in inflammatory bowel disease. Inflamm Bowel Dis 2007;13:993-1000.

50. Kim $\mathrm{P}$, Chung $\mathrm{E}$, Yamashita $\mathrm{H}$, et al. In vivo wide-area cellular imaging by side-view endomicroscopy. Nat Methods 2010;7:303-305. 